\title{
Arcwise Connected Domains, Quasiconformal Mappings, and Quasidisks
}

\author{
Yu-Ming Chu \\ School of Mathematics and Computation Sciences, Hunan City University, Yiyang 413000, China \\ Correspondence should be addressed to Yu-Ming Chu; chuyuming2005@126.com
}

Received 13 August 2014; Accepted 16 August 2014; Published 28 August 2014

Academic Editor: Zhi-Gang Wang

Copyright ( 2014 Yu-Ming Chu. This is an open access article distributed under the Creative Commons Attribution License, which permits unrestricted use, distribution, and reproduction in any medium, provided the original work is properly cited.

We prove that a homeomorphism $f: R^{2} \rightarrow R^{2}$ is a quasiconformal mapping if and only if $f(D)$ is an arcwise connected domain for any arcwise connected domain $D \subseteq R^{2}$, and $D$ is a quasidisk if and only if both $D$ and its exterior $D^{*}=R^{2} \backslash \bar{D}$ are arcwise connected domains.

\section{Introduction}

We shall assume throughout this paper that $D$ is a Jordan proper subdomain of the complex plane $R^{2}$ with a boundary containing at least three points. For convenience we shall adopt the notation and terminology as in paper [1]. For $x \in R^{2}$ and $0<r<\infty$, let $B^{2}(x, r)=\left\{z \in R^{2}\right.$ : $|z-x|<r\}, \bar{B}^{2}(x, r)$ be the closure of $B^{2}(x, r), S^{1}(x, r)=$ $\partial B^{2}(x, r), B^{2}(r)=B^{2}(0, r)$, and $B^{2}=B^{2}(1)$. Suppose that $f$ is a homeomorphism in $R^{2}, x \in R^{2}$ and $0<r<\infty$, let $L(x, f, r)=\max _{|y-x|=r}|f(y)-f(x)|$, and $l(x, f, r)=$ $\min _{|y-x|=r}|f(y)-f(x)|$.

Suppose that $b \geq 1$ is a constant, we say that $D$ is a $b$ arcwise connected domain if each pair of points $x$ and $y$ in $D$ can be joined by an $\operatorname{arc} \gamma \subseteq D$ with $\operatorname{dia}(\gamma) \leq b|x-y|$. Here $\operatorname{dia}(\gamma)$ is the Euclidean diameter of $\gamma$. And we call $D$ an arcwise connected domain if $D$ is a $b$-arcwise connected domain for some $b \geq 1$.

Arcwise connected domain is an important concept; it has been used extensively in the research fields of superspaces [2], multiobjective programmings [3], color images [4], continuous mappings [5], fixed points theory [6], continuity and differential problems [7], and topology groups theory [8].

$D$ is called a quasidisk if there exists a $K$-quasiconformal mapping $(K \geq 1) f: R^{2} \rightarrow R^{2}$ such that $D$ is the image of the unit disk $B^{2}$ under $f$.
It is well-known that quasidisks play a very important role in quasiconformal mappings, complex dynamics, Fuchsian groups, and Teichmüller space theory [9-12].

The purpose of this paper is to prove the following Theorems 1 and 2.

Theorem 1. A homeomorphism $f: R^{2} \rightarrow R^{2}$ is a quasiconformal mapping if and only if $f(D)$ is a arcwise connected domain for any arcwise connected domain $D \subseteq R^{2}$.

Theorem 2. $D$ is a quasidisk if and only if both $D$ and $D^{*}=$ $R^{2} \backslash \bar{D}$ are arcwise connected domains.

For convenience we shall introduce the following concepts and they will be used in the next section.

Let $c \geq 1$ be a constant. (1) If for any $x_{0} \in R^{2}$ and $0<$ $r<+\infty$, points in $D \cap \bar{B}\left(x_{0}, r\right)$ can be joined by an arc in $D \cap \bar{B}\left(x_{0}, c r\right)$; then we say that $D$ is a $c$-inner linearly locally connected domain, denoted by $D \in c$-ILC; (2) If for any $x_{0} \in$ $R^{2}$ and $0<r<+\infty$, points in $D \backslash B^{2}\left(x_{0}, r\right)$ can be joined by an arc in $D \backslash B^{2}\left(x_{0}, r / c\right)$; then we say that $D$ is a $c$-outer linearly locally connected domain, denoted by $D \in c$-OLC.

$D$ is said to be a linearly locally connected domain if there exists $c \geq 1$ such that $D \in c$-ILC and $D \in c$-OLC. 
Let $b>0$ be a constant; then $D$ is said to be a $b$-cigar domain if each pair of points $x_{1}, x_{2} \in D$ can be joined by an $\operatorname{arc} \gamma \subseteq D$ for which

$$
\min _{j=1,2} \operatorname{dia}\left(\gamma\left(x_{j}, x\right)\right) \leq b d(x, \partial D) \quad \forall x \in \gamma,
$$

where $\gamma\left(x_{j}, x\right)$ is the part of $\gamma$ between $x_{j}$ and $x$, and $d(x, \partial D)$ is the Euclidean distance from $x$ to $\partial D$.

We say that $D$ is a cigar domain if $D$ is a $b$-cigar domain for some $b>0$.

Gehring and Osgood proved the following result in [13].

Theorem A. $D$ is a quasidisk if and only if $D$ is a linearly locally connected domain.

\section{Proof of Theorems 1 and 2}

To prove our Theorems 1 and 2, we first establish and introduce several lemmas.

Lemma 3. If $D$ is an arcwise connected domain, then $D^{*}=$ $R^{2} \backslash \bar{D}$ is a cigar domain.

Proof. For any $z_{1}, z_{2} \in D^{*}$, let $\gamma$ be the hyperbolic geodesic joining $z_{1}$ and $z_{2}$ in $D^{*}$, for any $z \in \gamma \backslash\left\{z_{1}, z_{2}\right\}$; suppose that $f: B^{2} \rightarrow D^{*}$ is a conformal mapping with $f(0)=z$ and

$$
f^{-1}(\gamma) \subseteq R=\left\{z: z \in R^{2}, \operatorname{Im} z=0\right\},
$$

where $f^{-1}$ is the inverse of $f$. Then there exist

$$
x_{1} \in\left\{z: z \in S^{1}, \operatorname{Im} z>0\right\}, \quad x_{2} \in\left\{z: z \in S^{1}, \operatorname{Im} z<0\right\}
$$

by $[14$, Corollary 10.3$]$ such that $\alpha_{j}=f\left(\left[0, x_{j}\right)\right)$ is rectifiable with

$$
l\left(\alpha_{j}\right)<a_{0} d\left(z, \partial D^{*}\right), \quad j=1,2,
$$

where $a_{0}$ is an absolute constant, and $\left[0, x_{j}\right)$ is the half open segment joining the origin $O$ and $x_{j} ; j=1,2$.

Let $y_{j}=f\left(x_{j}\right), \alpha=\alpha_{1} \cup \alpha_{2}$, then $y_{j} \in \partial D$ and

$$
l(\alpha) \leq l\left(\alpha_{1}\right)+l\left(\alpha_{2}\right)<2 a_{0} d\left(z, \partial D^{*}\right) .
$$

For above $y_{1}, y_{2} \in \partial D^{*}=\partial D$, there exist a constant $b \geq 1$ and a simple curve $\beta \subseteq D$ joining $y_{1}$ and $y_{2}$ such that

$$
\operatorname{dia}(\beta) \leq b\left|y_{1}-y_{2}\right| \leq b l(\alpha)
$$

by $D$ is an arcwise connected domain.

If we denote by $D_{0}$ the bounded domain with boundary $\alpha \cup \beta$, then one of the points $z_{1}$ and $z_{2}$ must be in $D_{0}$. Without loss of generality, we may assume that $z_{1} \in D_{0}$; then it follows from (5) and (6) that

$$
\begin{aligned}
\operatorname{dia}\left(\gamma\left(z_{1}, z\right)\right) & \leq \operatorname{dia}\left(D_{0}\right)=\operatorname{dia}\left(\partial D_{0}\right) \\
& \leq \operatorname{dia}(\alpha)+\operatorname{dia}(\beta) \\
& \leq l(\alpha)+\operatorname{dia}(\beta) \\
& <2 a_{0}(b+1) d\left(z, \partial D^{*}\right) .
\end{aligned}
$$

Inequality (7) leads to

$$
\min _{j=1,2} \operatorname{dia}\left(\gamma\left(z_{j}, z\right)\right)<2 a_{0}(b+1) d\left(z, \partial D^{*}\right) .
$$

Therefore, $D^{*}$ is a cigar domain that follows from (8).

Lemma 4. If $D$ is a c-cigar domain, then $D \in(2 c+2)-O L C$.

Proof. Let $b=2 c+2$. If $D \notin b$-OLC, then there exist $y_{0} \in R^{2}$, $0<r<\infty$ and $x_{1}, x_{2} \in D \backslash B^{2}\left(y_{0}, r\right)$, such that $x_{1}$ and $x_{2}$ can not be joined by any arc in $D \backslash B^{2}\left(y_{0}, r / b\right)$.

Since $D$ is a $c$-cigar domain, there exists an $\operatorname{arc} \gamma \subset D$ such that $\gamma$ joining $x_{1}$ and $x_{2}$ with

$$
\min _{j=1,2} \operatorname{dia}\left(\gamma\left(x_{j}, x\right)\right) \leq c d(x, \partial D)
$$

for all $x \in \gamma$.

It is obvious that $\gamma \cap S^{1}\left(y_{0}, r / b\right) \neq \emptyset$. Let $y \in \gamma \cap S^{1}\left(y_{0}, r / b\right)$; then (9) implies

$$
\begin{aligned}
d(y, \partial D) & \geq \frac{1}{c} \min _{j=1,2} \operatorname{dia}\left(\gamma\left(x_{j}, y\right)\right) \geq \frac{1}{c} \min _{j=1,2}\left|x_{j}-y\right| \\
& \geq \frac{1}{c}\left(1-\frac{1}{b}\right) r .
\end{aligned}
$$

But

$$
d\left(y_{0}, \partial D\right) \leq \frac{r}{b}
$$

Inequalities (10) and (11) together with the triangular inequality yield

$$
\begin{aligned}
\frac{1}{c}\left(1-\frac{1}{b}\right) r \leq d(y, \partial D) & \leq\left|y-y_{0}\right|+d\left(y_{0}, \partial D\right) \leq \frac{2}{b} r, \\
b & \leq 2 c+1 .
\end{aligned}
$$

Inequality (12) contradicts with $b=2 c+2$. Hence $D \in(2 c+2)$ OLC.

Lemma 5. If $D^{*}$ is a $c_{0}$-cigar domain, then $D \in\left(16 c_{0}+21\right)$ ILC.

Proof. Let $\delta=8 c_{0}+10$. For any $u \in R^{2}, s>0$, and $z_{1}, z_{2} \in D \cap$ $\bar{B}^{2}(u, s), z_{1} \neq z_{2}$. Denote $z=(1 / 2)\left(z_{1}+z_{2}\right)$ and $r=\left|z_{1}-z_{2}\right|$. We first prove that $z_{1}, z_{2}$ must be in the same component of $\bar{B}^{2}(z,(1 / 2) \delta r) \backslash D^{*}$.

If $z_{1}, z_{2}$ belong to different components of $\bar{B}^{2}(z,(1 / 2) \delta r) \backslash$ $D^{*}$, then $z_{1}, z_{2}$ must be in the different components of $\bar{B}^{2}(z,(1 / 2) r) \backslash D^{*}$. Let $\beta$ be the line segment which joins $z_{1}$ and $z_{2}$; then $\beta$ contains a subcurve $\alpha \subseteq D^{*}$ such that $\alpha$ divides $D^{*}$ into $D_{1}$ and $D_{2}$, and $\operatorname{dia}\left(D_{j}\right) \geq(1 / 2) r(\delta-1), j=1,2$. This yields

$$
\min _{j=1,2} \operatorname{dia}\left(D_{j}\right) \geq \frac{1}{2} r(\delta-1) .
$$


For any $x \in \alpha$, if $D_{1} \nsubseteq B^{2}\left(x,\left(2 c_{0}+2\right) \operatorname{dia}(\alpha)\right)$ and $D_{2} \nsubseteq$ $B^{2}\left(x,\left(2 c_{0}+2\right) \operatorname{dia}(\alpha)\right)$, then take

$$
x_{j} \in D_{j} \backslash \bar{B}^{2}\left(x,\left(2 c_{0}+2\right) \operatorname{dia}(\alpha)\right), \quad j=1,2 .
$$

Since $D^{*}$ is a $c_{0}$-cigar domain, there exists an $\operatorname{arc} \gamma \subseteq D^{*}$ joining $x_{1}$ and $x_{2}$ with

$$
\min _{j=1,2} \operatorname{dia}\left(\gamma\left(x_{j}, w\right)\right) \leq c_{0} d\left(w, \partial D^{*}\right)
$$

for all $w \in \gamma$.

Let $y \in \gamma \cap S^{1}(x, \operatorname{dia}(\alpha))$; then

$$
\begin{aligned}
& \left|y-x_{j}\right| \geq\left(2 c_{0}+1\right) \operatorname{dia}(\alpha), \\
& \min _{j=1,2} \operatorname{dia}\left(\gamma\left(x_{j}, y\right)\right) \leq c_{0} d\left(y, \partial D^{*}\right), \\
& \quad j=1,2 .
\end{aligned}
$$

This implies

$$
d\left(y, \partial D^{*}\right) \geq \frac{2 c_{0}+1}{c_{0}} \operatorname{dia}(\alpha)
$$

But

$$
d\left(x, \partial D^{*}\right) \leq \operatorname{dia}(\alpha) .
$$

Inequalities (17) and (18) together with the triangular inequality yield

$$
\begin{aligned}
\frac{2 c_{0}+1}{c_{0}} \operatorname{dia}(\alpha) & \leq d\left(y, \partial D^{*}\right) \leq|y-x|+d\left(x, \partial D^{*}\right) \\
& \leq 2 \operatorname{dia}(\alpha),
\end{aligned}
$$

so

$$
\operatorname{dia}(\alpha) \leq 0 .
$$

This is obviously impossible. Hence $D_{1} \subseteq \bar{B}^{2}\left(x,\left(2 c_{0}+\right.\right.$ 2) $\operatorname{dia}(\alpha))$ or $D_{2} \subseteq \bar{B}^{2}\left(x,\left(2 c_{0}+2\right) \operatorname{dia}(\alpha)\right)$, and we obtain

$$
\min _{j=1,2} \operatorname{dia}\left(D_{j}\right) \leq 2\left(2 c_{0}+2\right) \operatorname{dia}(\alpha) .
$$

Inequalities (13) and (21) together with $\operatorname{dia}(\alpha) \leq r$ imply

$$
\delta \leq 8 c_{0}+9 .
$$

This contradicts with $\delta=8 c_{0}+10$. Hence $z_{1}, z_{2}$ must be in the same component of $\bar{B}^{2}(z,(1 / 2) \delta r) \backslash D^{*}$, and there exists an $\operatorname{arc} \gamma \subseteq D$ joining $z_{1}$ and $z_{2}$ with

$$
\operatorname{dia}(\gamma) \leq \delta r=\delta\left|z_{1}-z_{2}\right| \leq 2 \delta s .
$$

It follows from (23) that

$$
\begin{aligned}
\gamma & \subseteq D \cap \bar{B}^{2}(u, s+\operatorname{dia}(\gamma)) \subseteq D \cap \bar{B}^{2}(u,(2 \delta+1) s) \\
& =D \cap \bar{B}^{2}\left(u,\left(16 c_{0}+21\right) s\right) .
\end{aligned}
$$

Hence $D \in\left(16 c_{0}+21\right)$-ILC; this completes the proof of Lemma 5.
Lemma 6. If $D$ is a b-cigar domain, then $D^{*}$ is a $(4 b+3)$ arcwise connected domain.

Proof. For any $z_{1}, z_{2} \in D^{*}, z_{1} \neq z_{2}$, let $z_{0}=\left(z_{1}+z_{2}\right) / 2$, $r=\left|z_{1}-z_{2}\right| / 2$, and $d=4 b+3$. We shall prove that $z_{1}$ and $z_{2}$ can be joined by an arc in $D^{*} \cap \bar{B}^{2}\left(z_{0}, d r\right)$.

If $z_{1}$ and $z_{2}$ can not be joined by any arc in $D^{*} \cap \bar{B}^{2}\left(z_{0}, d r\right)$, then $z_{1}$ and $z_{2}$ must be in the different components of $D^{*} \cap$ $\bar{B}^{2}\left(z_{0}, d r\right)$, and hence $z_{1}$ and $z_{2}$ must be in the different components of $D^{*} \cap \bar{B}^{2}\left(z_{0},(d / 2) r\right)$. There exist points $y_{1}$, $y_{2} \in \partial D^{*} \backslash \bar{B}^{2}\left(z_{0},(d / 2) r\right)$ such that $\beta \cap B^{2}\left(z_{0}, r\right) \neq \emptyset$ for any $\operatorname{arc} \beta \subseteq D$ joining $y_{1}$ and $y_{2}$.

Since $D$ is a $b$-cigar domain, hence there exists an $\operatorname{arc} \gamma \subseteq$ $D$ joining $y_{1}$ and $y_{2}$ such that

$$
\min _{j=1,2} \operatorname{dia}\left(\gamma\left(y_{j}, y\right)\right) \leq b d(y, \partial D)
$$

for all $y \in \gamma$.

Let $y_{0} \in \gamma \cap B^{2}\left(z_{0}, r\right)$; then

$$
\min _{j=1,2} \operatorname{dia}\left(\gamma\left(y_{j}, y_{0}\right)\right) \leq b d\left(y_{0}, \partial D\right),
$$

$$
d\left(y_{0}, \partial D\right) \leq\left|y_{0}-z_{j}\right| \leq 2 r,
$$

$$
\begin{aligned}
\min _{j=1,2} \operatorname{dia}\left(\gamma\left(y_{j}, y_{0}\right)\right) & \geq \min _{j=1,2}\left|y_{j}-y_{0}\right| \\
& \geq \min _{j=1,2}\left(\left|y_{j}-z_{0}\right|-\left|y_{0}-z_{0}\right|\right) \\
& \geq\left(\frac{d}{2}-1\right) r .
\end{aligned}
$$

It follows from (26) and (27) that

$$
d \leq 4 b+2,
$$

which contradicts with $d=4 b+3$. Hence $z_{1}$ and $z_{2}$ can be joined by an $\operatorname{arc} \alpha \subseteq D^{*} \cap \bar{B}^{2}\left(z_{0}, d r\right)$ with

$$
\operatorname{dia}(\alpha) \leq 2 d r=(4 b+3)\left|z_{1}-z_{2}\right| .
$$

This shows that $D^{*}$ is a $(4 b+3)$-arcwise connected domain.

Lemma 7. If $f: R^{2} \rightarrow R^{2}$ is a K-quasiconformal mapping, and $c \geq 1$ is a constant, then for any $x \in R^{2}$ and $0<r<+\infty$, we have

$$
L\left(x, f, c L\left(x^{\prime}, f^{-1}, r\right)\right) \leq a r,
$$

where $x^{\prime}=f(x), f^{-1}$ is the inverse of $f$, and $a=a(K, c)$ is a constant which depends only on $K$ and $c$.

Proof. Let $\Gamma$ be the curve family which joins $S^{1}\left(x, c L\left(x^{\prime}\right.\right.$, $\left.\left.f^{-1}, r\right)\right)$ and $\partial\left(f^{-1}\left(B^{2}\left(x^{\prime}, r\right)\right)\right)$ in $B^{2}\left(x, c L\left(x^{\prime}, f^{-1}, r\right)\right)$ । $\overline{f^{-1}\left(B^{2}\left(x^{\prime}, r\right)\right)} ; M(\Gamma)$ denotes the modulus of $\Gamma, \Gamma^{\prime}=f(\Gamma)$. 
Then from the comparison principle of modulus and the result given in $[1,7.5]$ we get

$$
\begin{gathered}
M(\Gamma) \geq \frac{2 \pi}{\log \left(c L\left(x^{\prime}, f^{-1}, r\right) / l\left(x^{\prime}, f^{-1}, r\right)\right)}, \\
M\left(\Gamma^{\prime}\right) \leq \frac{2 \pi}{\log \left(l\left(x, f, c L\left(x^{\prime}, f^{-1}, r\right)\right) / r\right)} .
\end{gathered}
$$

According to the properties of $K$-quasiconformal mapping in [1], we have

$$
\begin{aligned}
& K M\left(\Gamma^{\prime}\right) \geq M(\Gamma) \geq \frac{M\left(\Gamma^{\prime}\right)}{K} \\
& \frac{L\left(x^{\prime}, f^{-1}, r\right)}{l\left(x^{\prime}, f^{-1}, r\right)} \leq c^{\prime}=c^{\prime}(K),
\end{aligned}
$$

where $c^{\prime}(K)$ is a constant which depends only on $K$.

It follows from (31)-(33) that

$$
\frac{l\left(x, f, c L\left(x^{\prime}, f^{-1}, r\right)\right)}{r} \leq\left(c c^{\prime}\right)^{K} .
$$

The same reason to get (33) implies

$$
\frac{L\left(x, f, c L\left(x^{\prime}, f^{-1}, r\right)\right)}{l\left(x, f, c L\left(x^{\prime}, f^{-1}, r\right)\right)} \leq c^{\prime} .
$$

It follows from (34) and (35) that

$$
\frac{L\left(x, f, c L\left(x^{\prime}, f^{-1}, r\right)\right)}{r} \leq c^{\prime}\left(c c^{\prime}\right)^{K}=a .
$$

Lemma 8. Suppose that $f: R^{2} \rightarrow R^{2}$ is a K-quasiconformal mapping. If $D$ is a $b$-cigar domain for some $b>0$, then $D^{\prime}=$ $f(D)$ is a $b^{\prime}$-cigar domain. In here $b^{\prime}=b^{\prime}(b, K)$ is a constant which depends only on $b$ and $K$.

Proof. For any points $y_{1}, y_{2} \in D^{\prime}$, let $x_{1}=f^{-1}\left(y_{1}\right), x_{2}=$ $f^{-1}\left(y_{2}\right)$. Since $D$ is a $b$-cigar domain, hence there exists an $\operatorname{arc} \gamma \subseteq D$ joining $x_{1}$ and $x_{2}$ such that $\min _{j=1,2} \operatorname{dia}\left(\gamma\left(x_{j}, x\right)\right) \leq$ $b d(x, \partial D)$ for all $x \in \gamma$. Let $\gamma^{\prime}=f(\gamma)$; then $\gamma^{\prime}$ is an arc joining $y_{1}$ and $y_{2}$ in $D^{\prime}$. For any $y \in \gamma^{\prime}$, let $x=f^{-1}(y)$; then $x \in \gamma$ with $\min _{j=1,2} \operatorname{dia}\left(\gamma\left(x_{j}, x\right)\right) \leq b d(x, \partial D)$.

Without loss of generality, we assume that $\operatorname{dia}\left(\gamma\left(x_{1}, x\right)\right) \leq b d(x, \partial D)$; then $\gamma\left(x_{1}, x\right) \subseteq \bar{B}^{2}(x, b d(x, \partial D))$ and $\gamma^{\prime}\left(y_{1}, y\right)=f\left(\gamma\left(x_{1}, x\right)\right) \subseteq \bar{B}^{2}(y, L(x, f, b d(x, \partial D)))$. From Lemma 7 and the fact $L(x, f, b d(x, \partial D)) \leq$ $L\left(x, f, b L\left(y, f^{-1}, d\left(y, \partial D^{\prime}\right)\right)\right)$ we know that there exists constant $b^{\prime}=b^{\prime}(b, K)$ which depends only on $b$ and $K$ such that $L(x, f, b d(x, \partial D)) \leq\left(b^{\prime} / 2\right) d\left(y, \partial D^{\prime}\right)$. This implies $\gamma^{\prime}\left(y_{1}, y\right) \subseteq \bar{B}^{2}\left(y,\left(b^{\prime} / 2\right) d\left(y, \partial D^{\prime}\right)\right)$. Therefore,

$$
\begin{gathered}
\operatorname{dia}\left(\gamma^{\prime}\left(y_{1}, y\right)\right) \leq b^{\prime} d\left(y, \partial D^{\prime}\right), \\
\min _{j=1,2} \operatorname{dia}\left(\gamma\left(y_{j}, y\right)\right) \leq b^{\prime} d\left(y, \partial D^{\prime}\right) .
\end{gathered}
$$

This shows that $D^{\prime}$ is a $b^{\prime}$-cigar domain.
Lemma 9 (see [15]). Let $f: R^{2} \rightarrow R^{2}$ be a homeomorphism. If there exists a constant $c>0$ such that

$$
\left[\operatorname{dia}\left(f\left(B^{2}(x, r)\right)\right)\right]^{2} \leq c \cdot m\left[f\left(B^{2}(x, r)\right)\right]
$$

for all $x \in R^{2}$ and $0<r<\infty$; then $f$ is a quasiconformal mapping, where $m\left[f\left(B^{2}(x, r)\right)\right]$ denotes the 2-dimensional Lebesgue measure of $f\left(B^{2}(x, r)\right)$.

Lemma 10. Let $f ; R^{2} \rightarrow R^{2}$ be a homeomorphism. If $f$ maps any cigar domain $D$ onto a cigar domain $D^{\prime}=f(D)$, then $f$ is a quasiconformal mapping.

Proof. For any $x \in R^{2}$ and $0<r<\infty$, choose $y \in B^{2}(x, r)$ such that

$$
\operatorname{dia}\left(f\left(B^{2}(x, r)\right)\right) \leq 3|f(y)-f(x)| .
$$

It is easy to see that $B^{2}(x, r)$ is a 1-cigar domain if we take $\gamma=\left[x_{1}, x\right] \cup\left[x_{2}, x\right]$ for any $x_{1}, x_{2} \in B^{2}(x, r)$, where $\left[x_{j}, x\right]$ denotes the closed line segment joining $x_{j}$ and $x, j=1,2$. By the assumption of Lemma 10 we know that there exists a constant $b>0$ such that $f\left(B^{2}(x, r)\right)$ is a $b$-cigar domain; hence there exists an $\operatorname{arc} \gamma \subseteq f\left(B^{2}(x, r)\right)$ joining $f(x)$ and $f(y)$ with

$$
\begin{aligned}
& \min \{\operatorname{dia}(\gamma(f(x), z)), \operatorname{dia}(\gamma(f(y), z))\} \\
& \leq b d\left(z, \partial f\left(B^{2}(x, r)\right)\right)
\end{aligned}
$$

for all $z \in \gamma$. If we choose $z_{0} \in \gamma$ such that $\operatorname{dia}\left(\gamma\left(f(x), z_{0}\right)\right)=$ $\operatorname{dia}\left(\gamma\left(f(y), z_{0}\right)\right)$, then (39) and (40) imply

$$
\begin{aligned}
\operatorname{dia}\left(z_{0}, \partial f\left(B^{2}(x, r)\right)\right) & \geq \frac{\operatorname{dia}\left(\gamma\left(f(x), z_{0}\right)\right)}{b} \\
& \geq \frac{\operatorname{dia}(\gamma(f(x), f(y)))}{2 b} \\
& \geq \frac{|f(x)-f(y)|}{2 b} \\
& \geq \frac{\operatorname{dia}\left(f\left(B^{2}(x, r)\right)\right)}{6 b} .
\end{aligned}
$$

This yields

$$
\begin{aligned}
& B^{2}\left(z_{0}, \frac{\operatorname{dia}\left(f\left(B^{2}(x, r)\right)\right)}{6 b}\right) \subseteq f\left(B^{2}(x, r)\right), \\
& \pi\left(\frac{\operatorname{dia}\left[f\left(B^{2}(x, r)\right)\right]}{6 b}\right)^{2} \leq m\left(f\left(B^{2}(x, r)\right)\right), \\
& {\left[\operatorname{dia}\left(f\left(B^{2}(x, r)\right)\right)\right]^{2} \leq \frac{36 b^{2}}{\pi} m\left(f\left(B^{2}(x, r)\right)\right) .}
\end{aligned}
$$

From the above argument and Lemma 9 we know that $f$ is a quasiconformal mapping. 
Proof of Theorem 1. Consider the following.

Necessity. For any quasiconformal mapping $f: R^{2} \rightarrow R^{2}$ and any arcwise connected domain $D \subseteq R^{2}$, we know that $D^{*}$ is a cigar domain by Lemma 3. This and Lemma 8 imply that $f\left(D^{*}\right)$ is a cigar domain, and then $D^{\prime}=f(D)=\left(f\left(D^{*}\right)\right)^{*}$ is an arcwise connected domain by Lemma 6 .

Sufficiency. To prove a homeomorphism $f: R^{2} \rightarrow R^{2}$ is a quasiconformal mapping, making use of Lemma 10, we need only to prove that $D^{\prime}=f(D)$ is a cigar domain for any cigar domain $D$. In fact, for any cigar domain $D$, Lemma 6 implies $D^{*}$ is an arcwise connected domain. From this and the condition of Theorem 1 we know that $f\left(D^{*}\right)$ is an arcwise connected domain; then from Lemma 3 we know that $\left(f\left(D^{*}\right)\right)^{*}=f(D)=D^{\prime}$ is a cigar domain.

Proof of Theorem 2. Consider the following.

Necessity. Let $D$ be a quasidisk; then there exists a quasiconformal mapping $f: R^{2} \rightarrow R^{2}$ such that $D=f\left(B^{2}\right)$.

(1) It is obvious that $B^{2}$ is a 1 -arcwise connected domain; then from Theorem 1 we know that $D=f\left(B^{2}\right)$ is an arcwise connected domain.

(2) Since $B^{2}$ is a 1-cigar domain, hence $\left(B^{2}\right)^{*}$ is an arcwise connected domain; then Theorem 1 implies that $f\left(\left(B^{2}\right)^{*}\right)=D^{*}$ is an arcwise connected domain.

Sufficiency. Suppose that both $D$ and $D^{*}$ are arcwise connected domains.

(1) Since $D$ is an arcwise connected domain, hence $D^{*}$ is a cigar domain by Lemma 3; then Lemma 5 implies that $D \in$ ILC.

(2) Since $D^{*}$ is an arcwise connected domain, hence $D$ is a cigar domain by Lemma 3; then Lemma 4 implies that $D \in$ OLC.

From the above (1) and (2) together with Theorem A we know that $D$ is a quasidisk.

\section{Conflict of Interests}

The author declares that there is no conflict of interests regarding the publication of this paper.

\section{Acknowledgments}

This research was supported by the Natural Science Foundation of China under Grants 61374086 and 11171307 and the Natural Science Foundation of Zhejiang Province under Grant LY13A010004.

\section{References}

[1] J. Väisälä, Lectures on n-Dimensional Quasiconformal Mappings, Lecture Notes in Mathematics, Vol. 229, Springer, Berlin, Germany, 1971.
[2] I. Lončar, "Arcwise connected continua and Whitney maps," Georgian Mathematical Journal, vol. 12, no. 2, pp. 321-330, 2005.

[3] E. K. Makarov and N. N. Rachkovski, "Efficient sets of convex compacta are arcwise connected," Journal of Optimization Theory and Applications, vol. 110, no. 1, pp. 159-172, 2001.

[4] S. McGuinness, "Colouring arcwise connected sets in the plane. I," Graphs and Combinatorics, vol. 16, no. 4, pp. 429-439, 2000.

[5] W. Kuperberg and A. Lelek, "Homotopy properties of pathwise connected continua," Polska Akademia Nauk. Fundamenta Mathematicae, vol. 92, no. 1, pp. 29-41, 1976.

[6] J. B. Fugate and L. Mohler, "Fixed point theorems for arcpreserving mappings of uniquely arcwise-connected continua," Proceedings of the American Mathematical Society, vol. 123, no. 10, pp. 3225-3231, 1995.

[7] T. W. Körner, "A dense arcwise connected set of critical points-molehills out of mountains," Journal of the London Mathematical Society, vol. 38, no. 3, pp. 442-452, 1988.

[8] S. N. Hudson, "On the topology and geometry of arcwise connected, finite-dimensional groups," Pacific Journal of Mathematics, vol. 82, no. 2, pp. 429-450, 1979.

[9] Z. M. Balogh, "Hausdorff dimension distribution of quasiconformal mappings on the Heisenberg group," Journal d'Analyse Mathématiques, vol. 83, pp. 289-312, 2001.

[10] D. Sullivan, "Quasiconformal homeomorphisms and dynamics. II. Structural stability implies hyperbolicity for Kleinian groups," Acta Mathematica, vol. 155, no. 3-4, pp. 243-260, 1985.

[11] A. F. Beardon, The Geometry of Discrete Groups, Springer, New York, NY, USA, 1982.

[12] O. Lehto, Univalent Functions and Teichmüller Spaces, vol. 109 of Graduate Texts in Mathematics, Springer, New York, NY, USA, 1986.

[13] F. W. Gehring and B. G. Osgood, "Uniform domains and the quasihyperbolic metric," Journal d'Analyse Mathématique, vol. 36, no. 1, pp. 50-74, 1979.

[14] C. Pommerenke, Univalent Functions, Vandenhoeck \& Ruprecht, Götingen, Germany, 1975.

[15] X. G. He, "Uniform domains and quasiconformal mappings," Chinese Annals of Mathematics A, vol. 13, pp. 66-69, 1992 (Chinese). 


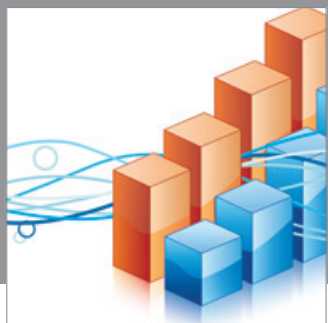

Advances in

Operations Research

mansans

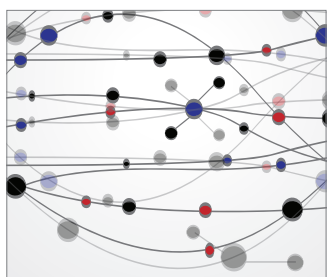

The Scientific World Journal
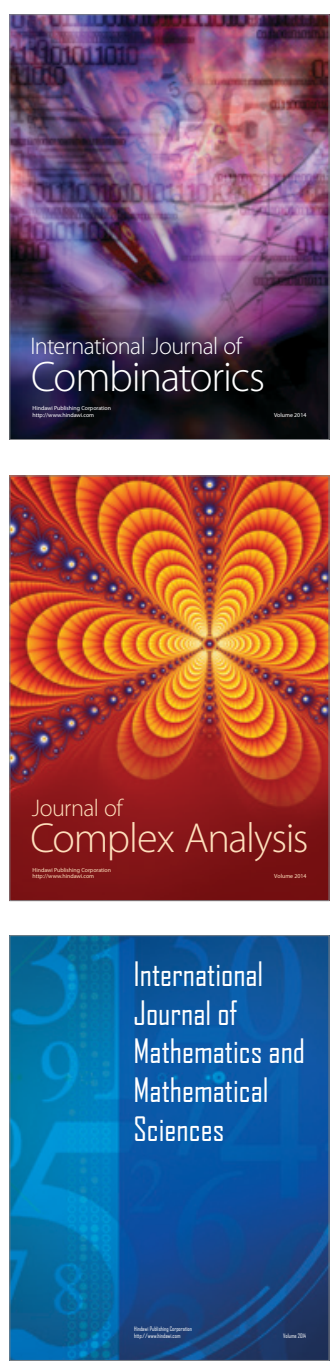
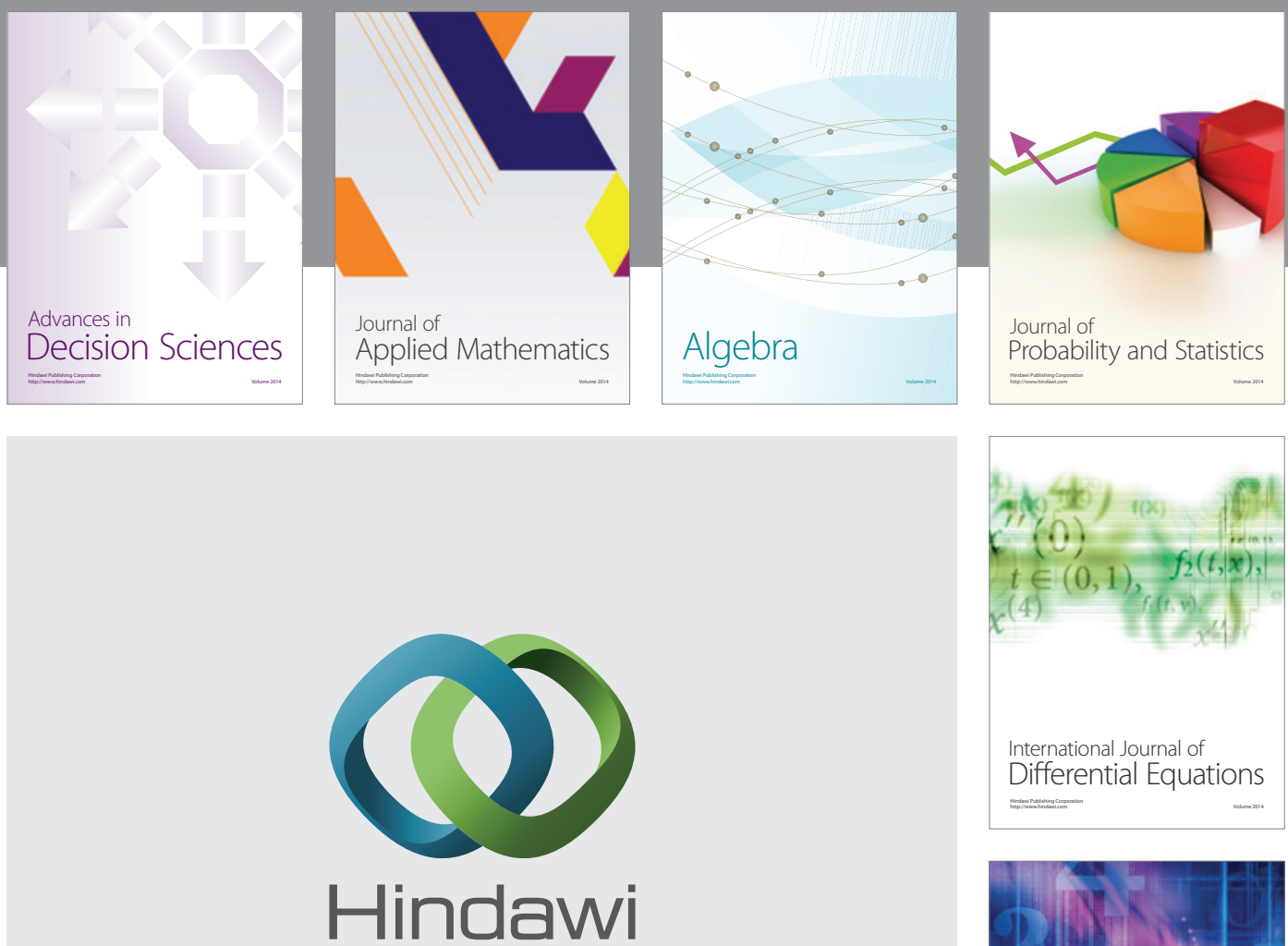

Submit your manuscripts at http://www.hindawi.com
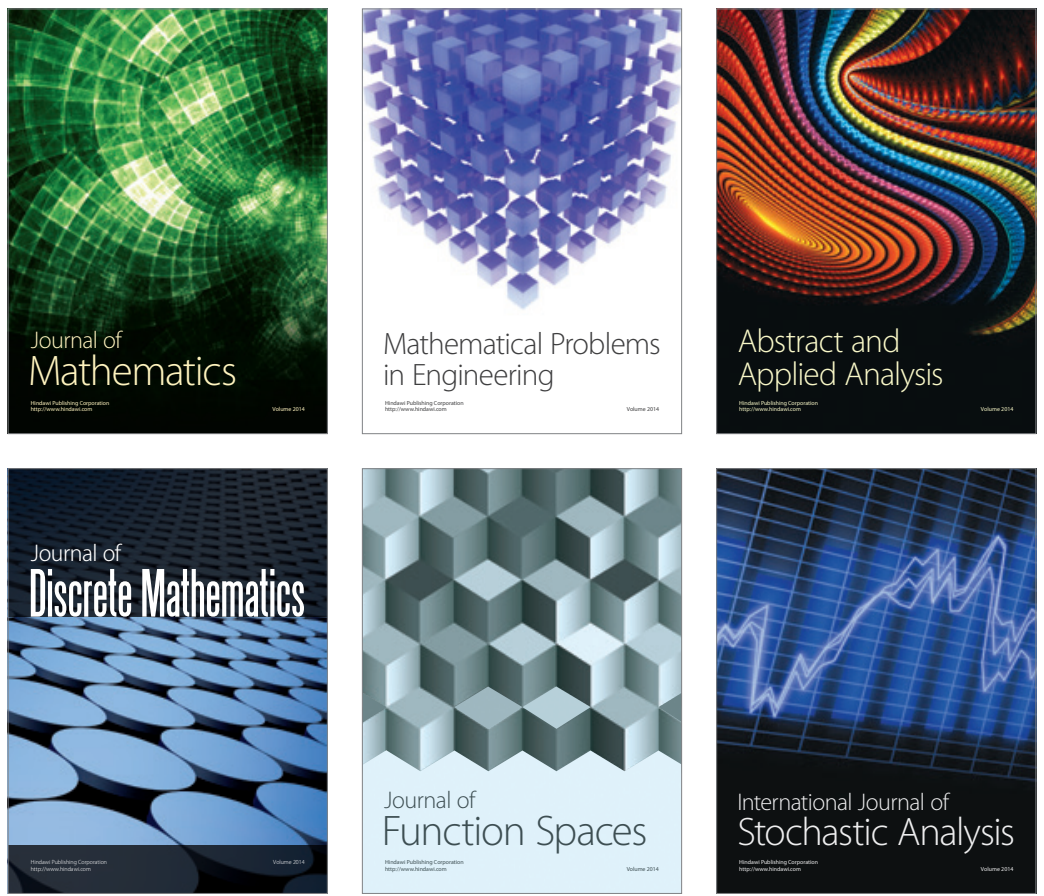

Journal of

Function Spaces

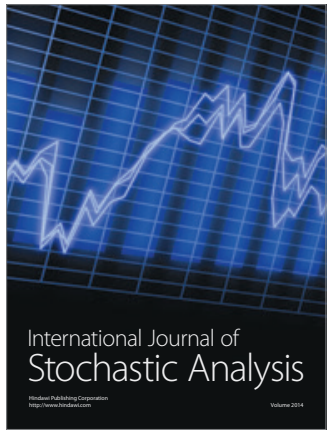

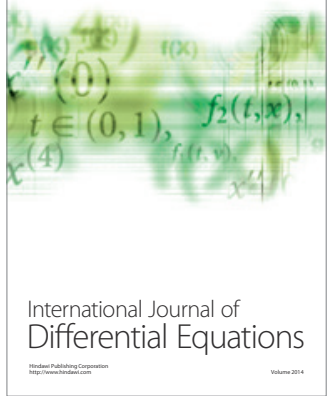
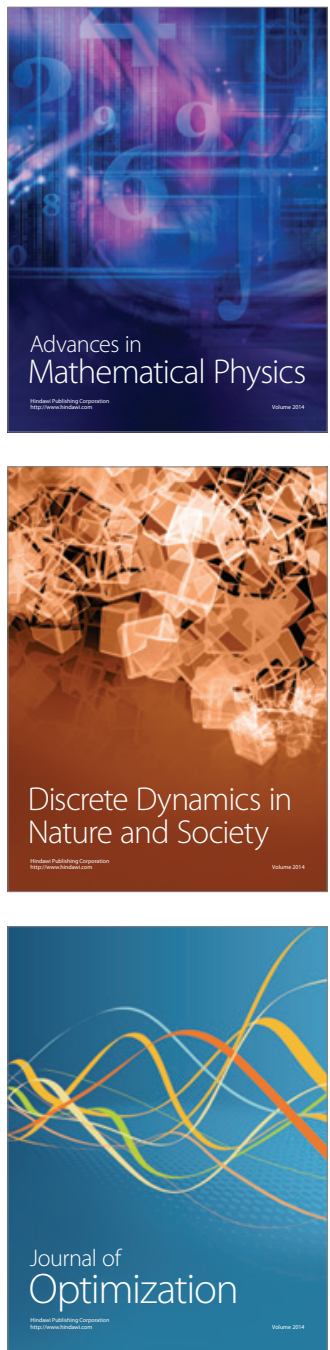